\title{
Design and Manufacture of Football Gates of Round Frame and Suspension
}

\author{
Guanghui Yang \\ Institute of Physical Education, Yanshan University, Hebei Province, 066004, China \\ ygh9116@ysu.edu.cn
}

\begin{abstract}
Keywords: Football; Gates; Round Frame; Suspension
Abstract. Exercising installations are playing important role in assisting the modern teaching and training of football, but so far few exercising installations have been developed. In this paper, novel football gates were designed and manufactured to improve the teaching and training of football, and the basic structure, working principle, processing, technical requirements, operation and application of the installations were described and investigated. With the help of the installations, the effects of teaching, training and game were markedly enhanced, and the popularization and development of football were given impetus to.
\end{abstract}

\section{Introduction}

The football gate is the key installation, which consists of frame, supports and net, although different sizes and styles of football gate in the teaching, training and game. There are two football gates of the same size, which are located at both ends of the football field. But, the gate has the following deficiencies. The front of the gate is the active area of shooting, and both sides are the dead. When exercising shooting, an athlete often shoots in flying or out of the bottom line, even though he stands within the active area, which leads athletes to frequently pick ball and dispel the enthusiasm. Especially in the case of more people and few ball, the training effect will be much worse. Besides, only in the front valid area, we are able to shoot with accuracy, which results few number of goals and weakens enjoyment. In addition, because of the gates installed in both ends of football field and only two teams in a football game, this traditional form of the game is monotonous and poor fun. To overcome these shortcomings, our group have designed and manufactured the gates of round frame and suspension, which can assist football techniques' teaching and training, make athletes quickly and effectively master the techniques and tactics, and promote the football's popularization and development.

\section{Designing the football gate of round frame}

Design philosophy. To assist technical training and innovate competitive mode, Our group has designed the gate of round frame to make athletes interest football and quickly master techniques. The frame is parallel to the ground, which is not only adopted in football technical and tactical training, but also change football competitive mode. With the help of the gate, the scoring rate is increased and the football's popularization and development is promoted. 


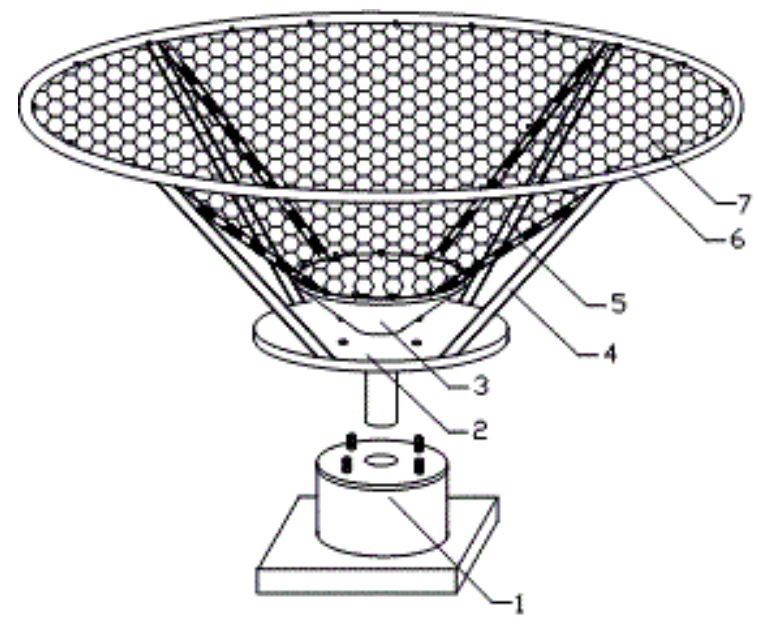

Fig. 1 Structure Diagram of the Football Gate of Round Frame

1-embedded part, 2-base plate, 3-truncated cone-shaped bracket, 4-outer support, 5-inner support, 6-round frame, 7-net

Processing and technical requirements. As can be seen from Fig.1, plug the lower end of the base plate into the jack of the upper end of the embedded part and they are fixed by screws and nuts. The upper end of the base plate is respectively connected to the lower ends of the four outer supports. And the the upper ends of the four outer supports are respectively connected to the lower end of round frame. The center of the base plate is connected to the lower end of the truncated cone-shaped bracket, the upper of which is connected to the lower ends of the four inner supports. And the upper ends of the four inner supports are connected to the lower end of the round frame. The frame, four inner supports and truncated cone-shaped bracket are respectively installed suspenders, which connect the net. And the embedded part has jack and four screws, and the base plate has four screw holes corresponding to the screws.

Applications. Build the football field into a circular, bury the embedded part in the center of the circular stadium, which is fixed by cement. Plug the lower end of the base plate into the jack of the upper end of the embedded part, and the four screws of the the embedded part are respectively plugged into the four screw holes of the base plate corresponding to the screws, which are fixed by nuts. Finally, hung the net. When the ball kicked into the frame and rolling along the net to the bottom, open the net which is hooked on the suspenders, remove the ball and hook the net. Athletes can practice the lob shot and also a variety of tactics according to the characteristics of the gate. We can formulate specific rules according to the characteristics of the gate. For example, two or four teams compete to kick ball into the frame in the center of the circular stadium. The result lies on the score a goal.

Beneficial Effects. The structure of the football gate of round frame is simple. The lower end of the embedded part is buried into the ground, which makes the gate more stable. Even if someone uses force to pull the frame, nor topple the gate. The frame is round, horizontal and up, which is propitious to practice lob shot at any angles. Athletes can practice lob shot around the gate, which is not only convenient to practice technique, but also to pick up ball. So, this football gate of round frame can increase the practical frequency of lob shot, shorten the picking time. Hence, it can improve the accuracy of lob shot and practical enthusiasm. We can also change competitive rules in accordance with the characteristics of the gate. That is to set up a football gate of round frame in the center of the football field, and two teams kick balls into the frame. In this way, it expands shooting range, increases goal difficulty and enhances the score rate, which makes the competition fiercer, more exciting and entertaining, and attracts more people to participate in football. 


\section{Designing the pensile football gates}

Design philosophy. In order to make the athletes more secure when they challenging for the ball in front of the football gate, the pensile football gates can be designed in the center of a football field, which has no post to effectively avoid sports injury. This gates can innovate the competitive form and be convenient to practice shooting, which enhance the football's entertainment and safety.

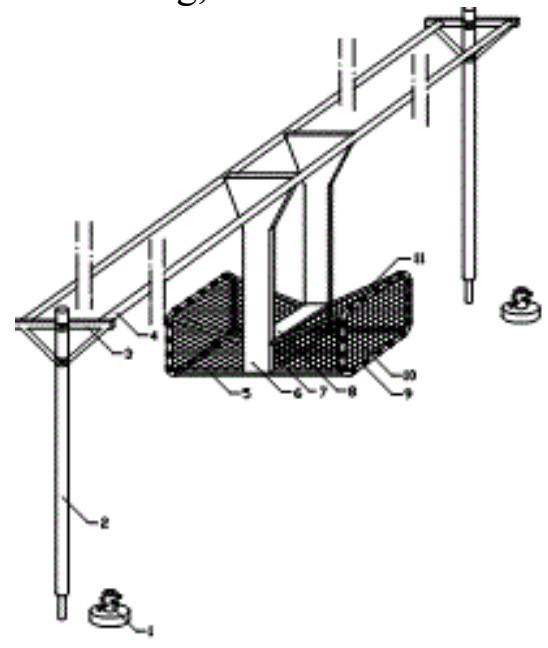

Fig. 2 Structure Diagram of the Pensile Football Gates

1-embedded part, 2-post, 3-tripod, 4-overarm, 5-lower support, 6-vertical wood, 7-lower crossbar, 8-upper crossbar, 9-frame, 10-net, 11-declining support

Processing and technical requirements. As can be seen from Fig.2, the lower ends of the two posts of the pensile football gates are respectively plugged into the circular holes of the two embedded parts, and the upper ends of the two posts are respectively connected to the two tripods by the screws and nuts. And the both ends of the two tripods are connected to the two overarms by the screws and nuts. The middle of the two overarms are respectively connected to the upper ends of the two vertical woods by the screws and nuts, the lower ends of which are respectively connected to the middle parts of the two lower supports. And the middle of the two lower supports and the lower crossbar form permanent joint, the middle of the two vertical woods and upper crossbar also form permanent joint. The both ends of the two lower supports are respectively connected to the lower ends of the two frames, the upper ends of which are respectively connected to the four declining supports. And the other ends of the four declining supports are respectively connected to the middle and lower parts of the two vertical woods. The frame, lower supports, vertical woods, lower crossbar, upper crossbar and declining supports are respectively installed suspenders, which connect the net.

Applications. Firstly, fix the two embedded parts out of the sideline of football field, then, insert the two posts into the circular holes of the two embedded parts, finally, install the two crossbars and gates, both of which are hung in the middle of football field. With this gates in teaching and training, we can specially arrange shooting, defensive techniques, running pass and so on. With this gates in entertainment game, we can formulate specific rules according to the characteristics of the field and gates. For example, either stipulate athletes to shoot to the opponent's gate, no offside limit and any moves, or any gate. In addition, make three teams or more to play football, which raises practitioners' interest and attracts more people to participate in the football.

Beneficial Effects. The structure of the pensile football gates is simple. The frame consists of the two posts and two crossbars, and the posts are fixed out of the sidelines. And the two gates are hung in the middle of the field. This gates can avoid the drawbacks that athletes easily hit the posts and improve the football safety. Because of the gates hung, it's convenient to challenge for the ball under or around the gates. Because of the gates in the middle of the field, it's convenient to shoot to the either gate in front of the gates. Athletes can pick up balls around the two gates, which increases shoot frequency and shortens picking time. Thus, this gates can mobilize the enthusiasm 
of the athletes, advance the practice effect and rapidly shooting techniques. Because of the two gates in the middle of football field, we can formulate specific rules according to the characteristics of the field and gates. Both players compete in the central area of football field, rapidly converse offense and defense, and increase shooting times, which makes the competition fiercer, more exciting and entertaining.

\section{Summary}

The football gates of round frame and suspension not only enrich the style of football gate, but also effectively assist the football techniques' training and game. And they make the corresponding traditional teaching and training more normative and scientific. Both the structures of the football gates are simple, safe and reliable. Experiments have shown that the football gates of round frame and suspension can increase the athletes' excitability and inspire their game interest, which make the game more intense, stormy and exciting, and diversify the item. The gates can satisfy both public and professional athletes' special requirements, so we can spread the gates in football.

\section{References}

[1]W. Liang, X.M. Liu: Journal of Sports Culture Guide Vol. 1 (2014), p. 151-153

[2]W. Zhang: Journal of Xian Sports College Vol. 5 (2014), p. 553-558

[3]P. Mao: Journal of Sports and Science Vol. 35 (2014), p. 77-79

[4]J. Cui, T.A. Zhang: Journal of Beijing Sports University Vol. 6 (2014), p. 120-124

[5]Y.L. Xie: Journal of Sports Culture Guide Vol. 2 (2010), p. 110-113

[6]Z.M. Hao, Q. Kang: Science Technology and Engineering Vol. 8 (2014), p. 36-41 